\title{
Exact solution approaches for bilevel lot-sizing
}

\author{
Tamás Kis ${ }^{\mathrm{a}, *}$, András Kovács ${ }^{\mathrm{a}, 1}$ \\ ${ }^{a}$ Computer and Automation Research Institute, Hungarian Academy of Sciences, H1111 \\ Budapest, Kende str. 13-17, Hungary
}

\begin{abstract}
In this paper we propose exact solution methods for a bilevel uncapacitated lotsizing problem with backlogs. This is an extension of the classical uncapacitated lot-sizing problem with backlogs, in which two autonomous and self-interested decision makers constitute a two-echelon supply chain. The leader buys items from the follower in order to meet external demand at lowest cost. The follower also tries to minimize its costs. Both parties may backlog. We study the leader's problem, i.e., how to determine supply requests over time to minimize its costs in view of the possible actions of the follower. We develop two mixed-integer linear programming reformulations, as well as cutting planes to cut off feasible, but suboptimal solutions. We compare the reformulations on a series of benchmark instances.
\end{abstract}

Keywords: Supply Chain Management, Bilevel Optimization, Integer Programming, Extended formulations

\section{Introduction}

While most of the operations research literature investigates lot-sizing models with a single decision maker, it is widely recognized that the lot-sizing decisions of autonomous partners in the supply chain mutually affect each other. Recently,

*Corresponding author. Tel.: +36 1 2796156; fax: +3614667503.

Email addresses: tamas.kis@sztaki.hu (Tamás Kis), akovacs@sztaki.hu (András Kovács) 
new approaches have been investigated to fill this gap: integrated models with the objective of minimizing the total cost by centralized planning [17], and coordination mechanisms for driving the self-interested partners towards optimal performance on the system level without giving up autonomy or data privacy [1, 7]. In this paper we study a new approach based on bilevel optimization.

Consider a two-level supply chain consisting of a producer (or buyer), and a supplier. The producer faces a time varying external demand, that it wants to serve at a minimum cost. In order to meet the demand, it requests raw material or components from the supplier. The supplier in turn optimizes its production with respect to the supply requests received. Both the producer and the supplier may backlog (deliver late) some of their demands at the expense of additional penalty costs (without penalties, both parties may delay deliveries arbitrarily). If the producer backlogs some of the demands, it pays a proportional penalty to its customer(s), which raises its costs. Likewise, if the supplier backlogs some of the supply requests, it must pay a proportional penalty to the producer (for a similar backlog penalty scheme, see [16]). In addition, if the supplier delivers late, the producer may be forced to deliver late as well, incurring high penalty costs, unless it raises its stock levels at the expense of higher holding costs. The producer can avoid some of the extra costs if it knows the cost structure of the supplier (this may be the case, e.g., if both parties are divisions of the same corporate unit, cf. [5]). Using this knowledge, the producer can compute the optimal production plan of the supplier for any choice of supply requests, from which its own costs can be derived. Therefore, the producer can choose the supply requests such that the optimal production plan of the supplier for those requests minimizes his costs, provided there is a computationally efficient way of doing this. The supplier solves an uncapacitated lot-sizing problem with backlogs (ULSB), where the demands (supply requests) are set by the producer. However, the producer's problem is more complex, since it has to determine the supply requests, while taking into account the economical considerations of the supplier. In this paper we focus on the optimization problem of the producer. 
The above problem can naturally be formulated as a bilevel optimization problem, where the producer acts as the leader, and the the supplier as the follower, for terminology see Section 2.2. The leader decides first, and sets problem parameters (in our case supply requests) for the follower. Subsequently, the follower (supplier) solves its optimization problem with the parameters (supply requests) set by the leader. However, the follower's optimal solution influences the objective function value, or even the feasibility of the leader's solution. Therefore, the leader has to carefully choose the parameters sent to the follower.

Main contributions. Our goal in this paper is to develop and evaluate mixedinteger linear programming (MIP) formulations for the bilevel lot-sizing problem which are solvable efficiently on medium-size instances using a standard solver. The proposed MIP formulations consist of two main parts: the first part models the leader's lot-sizing problem, while the second part describes the optimal solution(s) of the follower for the supply requests. For modeling the leader's lotsizing problem, we propose two alternative formulations: one based on the ULSB formulation in the original problem variables, and another using a facility location based extended formulation. As for expressing the optimal solutions of the follower, we use a primal along with the dual of an extended formulation of the ULSB with variable demands (corresponding to the supply requests). We also provide new bounds on variables, as well as inequalities to cut off suboptimal solutions of the uncapacitated lot-sizing problems with backlogs. To the best of our knowledge, this is the first attempt for modeling the bilevel lot-sizing problem as a MIP, and solving it efficiently by MIP techniques. Moreover, the modeling of the follower's optimality conditions using an extended formulation, and without referring to complementarity conditions [10] is new.

Structure of the paper. The related literature is surveyed in Section 2. The bilevel lot-sizing problem is formally defined in Section 3. The MIP models are presented in Section 4. New bounds on variables and cutting planes for strengthening the linear relaxation of the ULSB problem with variable demands are presented in 
Section 5. The approaches are assessed in computational experiments in Section 6, and conclusions are drawn in Section 7. Finally, the necessary background in lot-sizing with backlogs is recapitulated in Appendix A.

\section{Related literature}

\subsection{Lot-sizing}

Fundamental results on dynamic lot-sizing models were published in [31, 33]. These papers consider uncapacitated lot-sizing models where the deterministic, time varying demand is known in advance over a finite planning horizon. Over the past decades the basic models have been extended by production capacities and various side constraints, for an overview see e.g., [2, 21, 24]. Albeit dynamic programming is still the most efficient method for solving the tractable cases $[20,30,31,33]$, they have been complemented by linear programming formulations for describing the convex hull of feasible solutions, see e.g., $[3,4,15$, 22, 23, 25, 29]. In particular, Küçükyavuz and Pochet [15] have given a complete description of the feasible solutions of the uncapacitated lot-sizing problem with backlogs (ULSB) in the space of original problem variables. Many times, it is easier to work with extended formulations, when new variables and constraints are introduced to obtain the linear formulation. The modeling of various features in lot-sizing by mixed-integer programs (MIP) are investigated in e.g., [6, 9]. As further extensions, different lot-sizing and scheduling models, including smallbucket and large-bucket, discrete and continuous time formulations, as well as single- and multi-level models are presented in [12, 21]. A tight extended formulation is presented in [20] for uncapacitated two-level lot-sizing, and a formulation is derived for the multi-item, multi-client case.

\subsection{Bilevel programming}

Bilevel programming addresses decision and optimization problems whose outcome is determined by the interplay of two self-interested decision makers who decide sequentially. The first decision maker, or leader, is assumed to have 
a complete knowledge of the second decision maker's (follower) problem and parameters. Therefore, to optimize its own objective function, the leader must consider the response that it can expect from the follower. Unless the follower has a unique optimal solution for any possible parameter values set by the leader, the precise definition of a bilevel optimization problem involves a tie-breaking rule as well. Namely, in the optimistic case, if the follower has more than one optimal solutions for the same parameter values, it is assumed that the follower always selects an optimal solution which is the most advantageous for the leader. In contrast, in the pessimistic case, if the follower's optimal solution is not unique for some parameter values, the leader assumes that the least advantageous will be selected. In that case the leader sets the parameters of the follower such that the maximum value (over the optimal solutions of the follower with respect to the given parameters) of the leader's objective function is minimized (assuming the leader has a minimization problem).

The motivation for bilevel programming stems from economic game theory. In a two-player Stackelberg game two competing firms, the market leader and a follower company, for example a new entrant, produce equivalent goods. The firms decide their production quantities sequentially, which together determine the market price, with the aim of maximizing their own profit [28]. The basic modeling and solution techniques in bilevel programming are presented in [10]. A review of applicable solution methods for various classes of bilevel programs is given in [8], whereas reformulations of continuous bilevel optimization problems into single-level problems are discussed in [11]. A combinatorial perspective on bilevel problems is presented in [19].

\subsection{Related applications of bilevel programming}

In [5], Bard argued that centralized decision making and planning in large organizations is unrealistic in practice, and proposed bilevel optimization as an alternative, more suitable solution approach. As an application, Bard considered the setting of transfer prices among divisions of a corporate unit, by using (linear) bilevel programming. The upper level problem is that of the corporate unit, that 
wishes to set the internal transfer prices among the divisions in such a way that the local optimal decisions of the divisions coincide with the corporate optimum. [27] introduced a bilevel programming model to a production and distribution planning problem in a supply chain, where the follower's problem can be modeled by linear programs, whose parametric solutions can be computed efficiently. A similar production and distribution problem subject to uncertainties is formulated as a probabilistic bilevel problem in [26]. [32] investigates the problem of coordinated planning in a supply chain under hard service time requirements. The production planning problem of a pharmaceutical company with two machines and $n$ products is investigated in [18]. The leader is interested in minimizing the setup costs on the two machines incurred from the change of products between periods, and decides which product to produce on which machine, whereas the follower solves $n$ lot-sizing problems without backlogs connected by capacity constraints. The proposed heuristic method governs the assignment of lots to machines, and uses a MIP solver for solving the follower's problem exactly.

In [34], the robust lot-sizing problem with a minimax regret objective function is formulated as a bilevel optimization problem. The input of the problem consists of an interval of possible demand values for each time period of the planning horizon, and a set of production periods has to be fixed so that over all feasible realizations of the demands, the difference between the best solution achievable by production in the chosen production periods only, and the optimal solution value (with arbitrary production periods), is minimized. This problem can be formulated as a pessimistic bilevel optimization problem, where the leader seeks the best choice of production periods, while the follower tries to find a realization of demands giving the worst objective function values. This is in strong contrast to our model, where each level solves a different lot-sizing problem with backlogs.

The bilevel lot-sizing problem studied in the present paper has been already defined in [14]. In that paper, four approaches for solving the lot-sizing problems in a two-level supply chain were compared in terms of the incurred costs and the underlying assumptions, but the computation times were neglected. In fact, the 
method for solving the bilevel lot-sizing problem was not able to solve instances with a planning horizon of 20 time periods within one hour of computation time.

\section{Problem formulation}

We consider a supply chain that provides a single product to its customers. It consists of two decision makers, a leader and a follower. The leader faces a time varying deterministic external demand $d_{t}^{1}, t=1, \ldots, n$, over a discrete time horizon of $n$ time periods. To satisfy the external demand, the leader computes a supply request of $\delta_{t}, t=1, \ldots, n$, and sends it to the follower. The follower in turn solves a lot-sizing problem with demands $\delta_{t}$ set by the leader. It generates a production plan that specifies for each period $t$ the amount $x_{t}^{2}$ to be produced. In those time periods $t$ with $x_{t}^{2}>0$, a fixed cost of $f_{t}^{2}$ and a variable cost of $p_{t}^{2} x_{t}^{2}$ are incurred. The amount $x_{t}^{2}$ is used to serve the request $\delta_{t}$ along with backlogged requests from previous periods; and the remaining quantity, if any, is kept on stock to satisfy future requests. The associated marginal costs are $g_{t}^{2}$ for backlogging, and $h_{t}^{2}$ for holding stocks. Backlogging costs are paid as penalties for late delivery to the leader. In period $t$ the follower delivers a quantity of $x_{t}^{1} \geq 0$ to the leader. If the follower backlogs in period $t$, i.e., $r_{t}^{2}>0$, then $x_{t}^{1}=0$, i.e., no delivery occurs. On the other hand, if $r_{t}^{2}=0$, then the delivered quantity $x_{t}^{1}$ equals the sum of demand requests in the interval $\left[t^{\prime}+1, \ldots, t\right]$ where $t^{\prime}<t$ is the latest time period with $x_{t^{\prime}}^{1}>0$ if such a period exits, and $t^{\prime}=0$ otherwise. Therefore, $x_{t}^{1}$ and $r_{t}^{2}$ satisfy

$$
\left.\begin{array}{l}
r_{t}^{2}=\sum_{\tau=1}^{t}\left(\delta_{\tau}-x_{\tau}^{1}\right) \\
x_{t}^{1} r_{t}^{2}=0
\end{array}\right\} \text { for all } t=1, \ldots, n-1 .
$$

A delivery of $x_{t}^{1}>0$ incurs a fixed cost of $f_{t}^{1}$, and a variable cost of $p_{t}^{1} x_{t}^{1}$ at the leader. The goods are used to satisfy the demand $d_{t}^{1}$ along with backlogged demands of the leader, and the remaining quantity, if any, is kept on stock. The inventory holding costs and the backlogging costs of the leader are $h_{t}^{1}$ and $g_{t}^{1}$, respectively. All the demands must be satisfied by the end of the horizon, i.e., 
$\sum_{t=1}^{n} d_{t}^{1}=\sum_{t=1}^{n} \delta_{t}=\sum_{t=1}^{n} x_{t}^{1}=\sum_{t=1}^{n} x_{t}^{2}$. The main problem parameters are summarized below:

$n=$ number of periods of the planning horizon

$d_{t}^{1}=$ external demand of the leader

$p_{t}^{1}=$ marginal production costs of the leader

$h_{t}^{1}=$ marginal holding costs of the leader

$g_{t}^{1}=$ marginal backlogging costs of the leader

$f_{t}^{1}=$ fixed production cost of the leader

$p_{t}^{2}=$ marginal production costs of the follower

$h_{t}^{2}=$ marginal holding costs of the follower

$g_{t}^{2}=$ marginal backlogging costs of the follower

$f_{t}^{2}=$ fixed production cost of the follower

The decision variables of the leader and those of the follower are the following:

$x_{t}^{1} \geq 0:$ production quantity of the leader in period $t$

$s_{t}^{1} \geq 0$ : stock of the leader at the end of period $t$

$r_{t}^{1} \geq 0:$ backlog of the leader in period $t$

$y_{t}^{1} \in\{0,1\}$ : indicates leader receives supply in period $t$

$\delta_{t} \geq 0:$ supply requested by the leader in period $t$

$x_{t}^{2} \geq 0:$ production quantity of the follower in period $t$

$s_{t}^{2} \geq 0:$ stock of the follower at the end of period $t$ 
$r_{t}^{2} \geq 0:$ backlog of the follower in period $t$

$y_{t}^{2} \in\{0,1\}$ : indicates follower produces in period $t$

$\beta_{t}^{2} \in\{0,1\}$ : indicates follower backlogs in period $t$

Without loss of generality we assume that $f_{t}^{1}, f_{t}^{2} \geq 0$ for all $t$, and to ensure that the optima are finite, $h_{t}^{1}+g_{t}^{1} \geq 0$ and $h_{t}^{2}+g_{t}^{2} \geq 0$ for all $t=1, \ldots, n-1$ (cf. [22]).

The optimal solution of the follower depends on the quantities $\delta_{t}$ requested by the leader, while that of the leader heavily depends on the supply received from the follower. Therefore, the leader has to carefully choose the requests $\delta_{t}$. Clearly, this is a bilevel optimization problem. In the following we focus on the optimistic case only (cf. Section 2.2). We set up a mathematical program (Bilevel-LS) for modeling the decision problem of the leader.

$$
\operatorname{Minimize} \sum_{t=1}^{n}\left(p_{t}^{1} x_{t}^{1}+f_{t}^{1} y_{t}^{1}+h_{t}^{1} s_{t}^{1}+g_{t}^{1} r_{t}^{1}-g_{t}^{2} r_{t}^{2}\right)
$$

subject to

$$
\begin{array}{ll}
x_{t}^{1}+s_{t-1}^{1}-r_{t-1}^{1}=d_{t}^{1}+s_{t}^{1}-r_{t}^{1}, & t=1, \ldots, n \\
r_{t}^{2}=\sum_{\tau=1}^{t}\left(\delta_{\tau}-x_{\tau}^{1}\right), & t=1, \ldots, n \\
x_{t}^{1} \leq M y_{t}^{1}, & t=1, \ldots, n \\
x_{t}^{1} \leq M\left(1-\beta_{t}^{2}\right), & t=1, \ldots, n-1 \\
s_{0}^{1}=s_{n}^{1}=r_{0}^{1}=r_{n}^{1}=0, & t=1, \ldots, n \\
x_{t}^{1}, r_{t}^{1}, s_{t}^{1}, \delta_{t} \geq 0, & t=1, \ldots, n \\
y_{t}^{1} \in\{0,1\}, &
\end{array}
$$




$$
\left(\begin{array}{l}
y^{2} \\
x^{2} \\
s^{2} \\
r^{2} \\
\beta^{2}
\end{array}\right) \in \arg \min \left\{\sum_{t=1}^{n}\left(p_{t}^{2} x_{t}^{2}+f_{t}^{2} y_{t}^{2}+h_{t}^{2} s_{t}^{2}+g_{t}^{2} r_{t}^{2}\right) \mid(11)-(17)\right\}
$$

where

$$
\begin{array}{ll}
x_{t}^{2}+\left(s_{t-1}^{2}-r_{t-1}^{2}\right)=\delta_{t}+\left(s_{t}^{2}-r_{t}^{2}\right), & t=1, \ldots, n \\
x_{t}^{2} \leq M y_{t}^{2}, & t=1, \ldots, n \\
s_{0}^{2}=s_{n}^{2}=r_{0}^{2}=r_{n}^{2}=0, & \\
x_{t}^{2}, s_{t}^{2}, r_{t}^{2} \geq 0, & t=1, \ldots, n \\
y_{t}^{2} \in\{0,1\}, & t=1, \ldots, n \\
r_{t}^{2} \leq M \beta_{t}^{2}, & t=1, \ldots, n-1 \\
\beta_{t}^{2} \in\{0,1\} & t=1, \ldots, n-1 .
\end{array}
$$

In this formulation $M$ is a big constant with $M=\sum_{t=1}^{n} d_{t}^{1}$.

The objective is to minimize the leader's total cost minus the penalty received from the follower for backlogged supply to compensate for the increased costs of the leader [14]. (All our results would remain valid if the leader's objective function did not contain the compensation term.) The constraints (3)-(9) and (11)-(15) represent lot-sizing problems with backlogging with the additional constraints (4), (6) and (16). Namely, (4) connects the supply by the follower in period $t$ to the production of the leader in the same period, see equation (1), whereas by (6) and (16), the delivery is $x_{t}^{1}=0$ if the follower backlogs in period $t$. Moreover, condition (10) expresses that the leader chooses an optimal production plan of the follower with respect to $\delta$. If there are several optimal solutions for $\delta$, then the leader picks the most advantageous one for itself. 
Table 1: Optimal solution of a sample problem

\begin{tabular}{|l|rrrr|rrr|rrr|}
\hline$t$ & 1 & 2 & 3 & 4 & 5 & 6 & 7 & 8 & 9 & 10 \\
\hline \hline$d_{t}^{1}$ & 71 & 84 & 43 & 21 & 4 & 81 & 59 & 44 & 32 & 46 \\
\hline$\delta_{t}$ & 82 & 73 & 68 & & 42.72 & 39.77 & 57.51 & 55.46 & 21.93 & 44.61 \\
\hline$x_{t}^{1}$ & 82 & 73 & 68 & & & 82.49 & 57.51 & 55.46 & 21.93 & 44.61 \\
$s_{t}^{1}$ & 11 & & 25 & 4 & & 1.49 & & 11.46 & 1.39 & \\
$r_{t}^{1}$ & & & & & & & & & & \\
\hline \hline$x_{t}^{2}$ & 82 & 141 & & & & 140 & & 122 & & \\
$s_{t}^{2}$ & & 68 & & & & 57.51 & & 66.54 & 44.61 & \\
$r_{t}^{2}$ & & & & & 42.72 & & & & & \\
\hline
\end{tabular}

Illustrative example. There are $n=10$ time periods. The leader's fixed production cost, and the marginal production, holding and backlogging costs are $f^{1}=100$, $p^{1}=1, h^{1}=6$, and $g^{1}=18$, respectively, in all periods. The cost parameters of the follower are $f^{2}=492, p^{2}=1, h^{2}=5$, and $g^{2}=6$. Table 1 presents an optimal solution for this instance of the bilevel lot-sizing problem. The columns of the table are indexed by the time periods 1 through 10 . The rows depict the external demand $d_{t}^{1}$, the supply requests sent to the follower $\delta_{t}$, the supply received from the follower $x_{t}^{1}$, and the stocking and backlogging quantities, $s_{t}^{1}$ and $r_{t}^{1}$, respectively, of the leader; and the production plan $x_{t}^{2}$, the stocking and backlogging quantities, $s_{t}^{2}$ and $r_{t}^{2}$, respectively, of the follower. Notice that the leader never backlogs in this example. Moreover, the external demand $d_{t}^{1}$ in periods $t=2,7,9$ is satisfied partly from production and partly from stock, i.e., $s_{t-1}^{1} x_{t}^{1}>0$. This distinguishes the leader's problem from the uncapacitated lot-sizing problem, which always admits an optimal solution such that the demand of a period is uniquely served either from production, from stock, or from backlog.

In addition to determining its own lot-sizes, the leader also controls the production of the follower via the supply requests $\delta_{t}$. The optimal values of the supply requests may deviate both from the external demand $d_{t}^{1}$ and the anticipated supply $x_{t}^{1}$. For instance, in time period 1, the leader inflates demand (requests 82 units 
instead of the external demand of 71). This is reasonable because a supply request less than 82 would cause backlog later at both levels. On the other hand, in period 5 , the leader requests a supply of 42.72 units that it will need only later, in period 6. This quantity does not trigger production at the follower, since it is more economical to pay some backlog costs instead of satisfying the request on time. Hence, the leader's benefit is the extra backlog compensation obtained from the follower.

The benefits of moving some of the demands earlier by the leader become obvious by comparison to a solution in which the leader does not move any demands earlier. This latter solution would incur higher backlogging costs paid to the customers, and less backlog compensations received from the follower, and yields a significantly higher solution value of 1571.14 instead of the value 869.639 of the optimal solution depicted in Table 1.

This example also illustrates the optimistic aspect of the problem. Namely, in period 1, the producer requests 82 units. The supplier has a choice between producing it in period 1 , or satisfying it from backlog. The associated cost in the former case is the setup cost plus the variable cost of production, which is $f^{2}+82 p^{2}=492+82=574$, whereas if the demand were satisfied from backlog from period 2, then the cost is again $82\left(g^{2}+p^{2}\right)=82(6+1)=574$. Hence, the supplier admits at least two optimal solutions. Since the supplier chooses the most advantageous one for the producer by assumption, it will backlog.

To avoid pathological cases, we want to ensure that $r_{t}^{2} s_{t}^{2}=0$ in any optimal solution of the follower. One way to achieve this is to add new constraints $s_{t}^{2} \leq$ $M\left(1-\beta_{t}^{2}\right), t=1, \ldots, n-1$, to the follower's program, but this would increase the number of constraints by $n-1$. Alternatively, noticing that a solution with $s_{t}^{2} r_{t}^{2}>0$ cannot be optimal for the follower unless $g_{t}^{2}+h_{t}^{2}=0$, we make the following:

Assumption 1. The backlogging and holding costs of the follower satisfy $g_{t}^{2}+h_{t}^{2}>$ 0 for $t=1, \ldots, n-1$. 


\section{MIP models}

In this section we describe two mixed-integer linear programs for solving bilevel lot-sizing problems. In both MIPs we introduce new variables and constraints to describe the connection between the supply requests $\delta_{t}$ and the corresponding optimal solutions of the follower. The two MIPs differ in modeling the leader's constraints.

\subsection{Formulation $M I P-N$}

Our first formulation is based on a MIP model whose feasible solutions are those $\left(x^{2}, y^{2}, s^{2}, r^{2}, \delta\right)$ vectors such that $\left(x^{2}, y^{2}, s^{2}, r^{2}\right)$ is an optimal solution of the follower's ULSB problem with demands (supply requests) $\delta_{t} \geq 0$ such that $\sum_{t=1}^{n} \delta_{t}=K$, where $K=\sum_{t=1}^{n} d_{t}^{1}$ is a fixed constant. Let $O P^{2}$ be the (mixedinteger) set of these vectors. Firstly, we give an extended formulation for $O P^{2}$, and then we will use it in our first formulation for the bilevel lot-sizing problem.

To get an extended formulation for $O P^{2}$, we express the optimality conditions of the follower by connecting a primal-, and the dual of an extended formulation of ULSB by a single constraint. As primal formulation, we choose the objective function in (10) subject to the constraints (11)-(17). Let $Z^{U L S B}(\delta)$ denote the optimum value of the follower for supply requests $\delta \geq 0$. As for the dual, we need a linear program whose feasible solutions provide lower bounds on $Z^{U L S B}(\delta)$, its optimum value is $Z^{U L S B}(\delta)$ for any $\delta \geq 0$, and the $\delta_{t}$ occur in the right hand side only. To get such a linear formulation, we start out from the shortest path formulation (A.7)-(A.8) of Appendix A in which the demand occurs in the objective function. Notice that this linear program always has a finite optimum for any fixed $\delta \geq 0$. Since $f_{t}^{2} \geq 0$, there always exists an optimal solution with $z_{t t}=y_{t}$. Hence, $z_{t t}$ can be substituted out. Taking the dual of the resulting linear program, the $\delta_{t}$ occur only in the right hand side of the constraints. The dual variables are $\phi_{t}^{2}, \phi_{t^{\prime}}^{2}$, and $\phi_{t^{\prime \prime}}^{2}$ for $t=1, \ldots, n$, and to simplify notation we define $\phi_{n+1}^{2}=0$.

$$
D^{S P}(\delta)=\max \phi_{1}^{2}
$$


subject to

$$
\left.\begin{array}{ll}
\phi_{t}^{2}-\phi_{k^{\prime}}^{2} \leq a_{k, t}^{2}, & k=t, \ldots, n \\
\phi_{t^{\prime}}^{2}-\phi_{t^{\prime \prime}}^{2} \leq p_{t}^{2} \delta_{t}+f_{t}^{2}, & \\
\phi_{t^{\prime \prime}}^{2}-\phi_{k+1}^{2} \leq b_{t, k}^{2}, & k=t, \ldots, n
\end{array}\right\} \text { for all } t=1, \ldots, n,
$$

where the terms $a_{k, \ell}^{2}$ and $b_{\ell, k}^{2}$ are defined in Lemma 6 of Appendix A, (with $p_{k}, g_{t}$ and $h_{t}$ substituted by $p_{k}^{2}, g_{t}^{2}$ and $h_{t}^{2}$, respectively). Moreover, by the strong duality of linear programming we have $Z^{U L S B}(\delta)=D^{S P}(\delta)$ for any fixed $\delta \geq 0$. We have the following:

Lemma 1. $\left(\hat{x}^{2}, \hat{y}^{2}, \hat{s}^{2}, \hat{r}^{2}, \hat{\delta}\right) \in O P^{2}$ if and only if $\sum_{t=1}^{n} \delta_{t}=K$, and there exists $\hat{\phi}^{2}, \hat{\beta}^{2}$ such that $\left(\hat{x}^{2}, \hat{y}^{2}, \hat{s}^{2}, \hat{r}^{2}, \hat{\beta}^{2}, \hat{\delta}, \hat{\phi}^{2}\right)$ satisfies the constraints (11)-(17), (19), and the equation

$$
\sum_{t=1}^{n}\left(p_{t}^{2} x_{t}^{2}+f_{t}^{2} y_{t}^{2}+h_{t}^{2} s_{t}^{2}+g_{t}^{2} r_{t}^{2}\right)=\phi_{1}^{2}
$$

Proof. First suppose $\left(\hat{x}^{2}, \hat{y}^{2}, \hat{s}^{2}, \hat{r}^{2}, \hat{\delta}\right) \in O P^{2}$. Let $\hat{\phi}^{2}$ denote an optimal solution of the dual linear program (18)-(19), and let $\hat{\beta}_{t}^{2}=1$ if $\hat{r}_{t}^{2}>0$, and 0 otherwise. Since $\left(\hat{x}^{2}, \hat{y}^{2}, \hat{s}^{2}, \hat{r}^{2}\right)$ is an optimal solution of the ULSB problem with respect to $\hat{\delta}$, we have $Z^{U L S B}(\hat{\delta})=D^{S P}(\hat{\delta})$. Hence, $\left(\hat{x}^{2}, \hat{y}^{2}, \hat{s}^{2}, \hat{r}^{2}, \hat{\beta}^{2}, \hat{\delta}, \hat{\phi}^{2}\right)$ satisfies all of the constraints (11)-(17), (19), and (20).

Conversely, suppose $\left(\hat{x}^{2}, \hat{y}^{2}, \hat{s}^{2}, \hat{r}^{2}, \hat{\beta}^{2}, \hat{\delta}, \hat{\phi}^{2}\right)$ satisfies all of the constraints (11)(17), (19), (20) and $\sum_{t=1}^{n} \delta_{t}=K$. Clearly, $\left(\hat{x}^{2}, \hat{y}^{2}, \hat{s}^{2}, \hat{r}^{2}\right)$ is a feasible solution of the follower's problem of value $\hat{\phi}_{1}^{2}$, since it satisfies (20). Therefore, we have $Z^{U L S B}(\delta) \leq D^{S P}(\delta)=Z^{U L S B}(\delta)$, where the first inequality follows from (20). Hence, $\left(\hat{x}^{2}, \hat{y}^{2}, \hat{s}^{2}, \hat{r}^{2}\right)$ is an optimal solution of the follower's ULSB for demand requests $\hat{\delta}$, and the statement follows.

Let $O P_{e x t}^{2}$ be the set of those $\left(x^{2}, y^{2}, r^{2}, s^{2}, \beta^{2}, \delta, \phi^{2}\right)$ vectors that satisfy the constraints (11)-(17), (19), (20), and $\sum_{t=1}^{n} \delta_{t}=K$.

Now we are ready to describe our first MIP for solving the bilevel lot-sizing 
problem.

$$
\text { MIP-N : } \min \left\{\begin{array}{l|l}
\sum_{t=1}^{n}\left(p_{t}^{1} x_{t}^{1}+f_{t}^{1} y_{t}^{1}+h_{t}^{1} s_{t}^{1}+g_{t}^{1} r_{t}^{1}-g_{t}^{2} r_{t}^{2}\right) & \begin{array}{l}
(3)-(9), \\
(11)-(17), \\
(19),(20)
\end{array}
\end{array}\right\}
$$

We can easily project any feasible solution of MIP-N to a solution of the bilevellot-sizing problem by discarding the values of variables $\phi^{2}$.

Lemma 2. There is the following correspondence between the feasible solutions of the bilevel lot-sizing problem and that of MIP-N:

(i) Any feasible solution of MIP-N can be projected into a feasible solution of the bilevel lot-sizing problem of the same value.

(ii) Conversely, any feasible solution of the bilevel lot-sizing problem can be extended to a feasible solution of MIP-N of the same value.

Proof. (i) Let $\left(\bar{x}^{1}, \bar{y}^{1}, \bar{s}^{1}, \bar{r}^{1}, \bar{\delta}, \bar{x}^{2}, \bar{y}^{2}, \bar{s}^{2}, \bar{r}^{2}, \bar{\beta}^{2}, \bar{\phi}^{2}\right)$ be a feasible solution of MIP$\mathrm{N}$. Firstly, we have to verify that $\left(\bar{x}^{2}, \bar{y}^{2}, \bar{s}^{2}, \bar{r}^{2}\right)$ is an optimal solution of the follower with respect to $\bar{\delta}$. Note that (3), (4) and (7) imply $\sum_{t=1}^{n} d_{t}^{1}=$ $\sum_{t=1}^{n} \bar{x}_{t}^{1}=\sum_{t=1}^{n} \bar{\delta}_{t}$, i.e., $K=\sum_{t=1}^{n} d_{t}^{1}$ in the definition of $O P_{\text {ext }}^{2}$. Since $\left(\bar{x}^{2}, \bar{y}^{2}, \bar{s}^{2}\right.$, $\left.\bar{r}^{2}, \bar{\beta}^{2}, \bar{\delta}, \bar{\phi}^{2}\right)$ satisfies the conditions of Lemma $1,\left(\bar{x}^{2}, \bar{y}^{2}, \bar{s}^{2}, \bar{r}^{2}\right)$ is an optimal solution of the follower for demand $\bar{\delta}$. Therefore, $\left(\bar{x}^{1}, \bar{y}^{1}, \bar{s}^{1}, \bar{r}^{1}, \bar{\delta}, \bar{x}^{2}, \bar{y}^{2}, \bar{s}^{2}, \bar{r}^{2}\right.$, $\bar{\beta}^{2}$ ) is a feasible solution of Bilevel-LS of the same objective function value as that of MIP-N.

(ii) Given a feasible solution $\left(\bar{x}^{1}, \bar{y}^{1}, \bar{s}^{1}, \bar{r}^{1}, \bar{\delta}, \bar{x}^{2}, \bar{y}^{2}, \bar{s}^{2}, \bar{r}^{2}, \bar{\beta}^{2}\right)$ of Bilevel-LS, since it is optimal for the follower, $\left(\bar{x}^{2}, \bar{y}^{2}, \bar{s}^{2}, \bar{r}^{2}\right)$ is an optimal solution of the follower's ULSB problem with respect to demands $\bar{\delta}_{t}$. Therefore, there exists a solution $\bar{\phi}^{2}$ of (19) which satisfies (20). Hence, $\left(\bar{x}^{1}, \bar{y}^{1}, \bar{s}^{1}, \bar{r}^{1}, \bar{\delta}, \bar{x}^{2}, \bar{y}^{2}, \bar{s}^{2}, \bar{r}^{2}, \bar{\beta}^{2}, \bar{\phi}^{2}\right)$ is a feasible solution of MIP-N. Clearly, the value of the solution of BilevelLS, and that of the corresponding solution of MIP-N are the same. 
Theorem 1. The bilevel lot-sizing problem always has a finite optimum, and the optimum value is that of MIP-N.

Proof. We argue that MIP-N always has a finite optimum. The statement then follows from Lemma 2. Clearly, the objective function value is bounded from below by $-M \sum_{t=1}^{n}\left(\left(p_{t}^{1}\right)^{-}+\left(h_{t}^{1}\right)^{-}+\left(g_{t}^{1}\right)^{-}+\left(g_{t}^{2}\right)^{+}\right)$, where $(v)^{-}=-\min \{0, v\}$, and $(v)^{+}=\max \{0, v\}$ (this is a very rough estimation). So, it suffices to prove that MIP-N has at least one feasible solution. Fix $\delta \geq 0$ arbitrarily such that $\sum_{t=1}^{n} \delta_{t}=$ $\sum_{t=1}^{n} d_{t}^{1}$. By Proposition 2, the follower has at least one optimal solution. Thus we can fix the values of variables $y_{t}^{2}, \beta_{t}^{2}$, as well as the values of variables $x_{t}^{2}, s_{t}^{2}, r_{t}^{2}$, and $\phi^{2}$. However, the leader's variables can also be fixed with respect to $\delta_{t}$ and $\beta_{t}^{2}$ in an obvious manner, thus a feasible solution is readily available.

\subsection{Formulation $M I P-F$}

The following formulation is a variant of MIP-N, in which the leader's lotsizing problem is modeled by the facility location based reformulation of ULSB, cf. Lemma 7 of Appendix A. The leader's constraints are (4), (6), and

$$
\begin{array}{ll}
x_{k}^{1}=\sum_{t=1}^{n} z_{k, t}^{1}, & \text { for all } k \\
\sum_{k=1}^{n} z_{k t}^{1}=d_{t}, & \text { for all } t \\
z_{k t}^{1}-d_{t} y_{k}^{1} \leq 0, & \text { for all } k, t \\
z_{k t}^{1} \geq 0, y_{k}^{1} \in\{0,1\}, & \text { for all } k, t .
\end{array}
$$

Then the facility location based reformulation is

$$
\text { MIP-F : } \min \left\{\begin{array}{l|l}
\sum_{k=1}^{n} \sum_{t=1}^{n} q_{k t}^{1} z_{k t}^{1}+\sum_{t=1}^{n} f_{t}^{1} y_{t}^{1} & \begin{array}{l}
(4),(6),(22), \\
(11)-(17), \\
(19),(20)
\end{array}
\end{array}\right\}
$$

where $q_{k t}^{1}=\left(p_{k}^{1}+h_{k}^{1}+\cdots h_{t-1}^{1}\right)$ if $k \leq t$ and $q_{k t}^{1}=\left(p_{k}^{1}+g_{t}^{1}+\cdots g_{k-1}^{1}\right)$ if $k>t$. One may verify that MIP-F is also a reformulation of Bilevel-LS, the details are omitted. 


\section{Valid inequalities on the optimal solutions of ULSB}

In this section we derive valid inequalities for the extended formulation of $O P^{2}$ (cf. Section 4.1). Since our results are valid for ULSB in general, we will omit the superscript 2. The following lemma enables us to apply the bounds and cuts developed in this section to the follower's ULSB problem.

Lemma 3. The bilevel lot-sizing problem always admits an optimal solution in which the follower has an extreme point solution of ULSB.

Proof. We will prove that any optimal solution of Bilevel-LS can be transformed into one with $r_{t}^{2} s_{t}^{2}=0, x_{t}^{2} s_{t-1}^{2}=0, x_{t}^{2} r_{t}^{2}=0$, and $s_{t-1}^{2} r_{t}^{2}=0$ for $t=1, \ldots, n$. Notice that $r_{n}^{2}=s_{n}^{2}=0$ by (13) in any feasible solution, so for $t=n$ we only have to verify $x_{t}^{2} s_{t-1}^{2}=0$.

Since $g_{t}^{2}+h_{t}^{2}>0, r_{t}^{2} s_{t}^{2}=0$ in any optimal solution of the follower, for $t=$ $1, \ldots, n-1$. Now we prove $x_{t}^{2} r_{t}^{2}=0$ and $s_{t-1}^{2} r_{t}^{2}=0$ for all $t$. Namely, rearranging (11) gives $x_{t}^{2}+s_{t-1}^{2}=\delta_{t}+r_{t-1}^{2}-r_{t}^{2}+s_{t}^{2}$. Suppose $r_{t}^{2}>0$, then by the previous point, $s_{t}^{2}=0$, and $x_{t}^{1}=0$ by (6). Since $x_{t}^{1}=\delta_{t}+r_{t-1}^{2}-r_{t}^{2}$ by (4), we have $x_{t}^{2}+s_{t-1}^{2}=\delta_{t}+r_{t-1}^{2}-r_{t}^{2}=x_{t}^{1}=0$. Since $x_{t}^{2}, s_{t-1}^{2} \geq 0$, the claim follows.

Finally, we prove that the optimal solution of the follower can be transformed such that $s_{t-1}^{2} x_{t}^{2}=0$ for all $t$, while maintaining $r_{t}^{2} s_{t}^{2}=0, s_{t-1}^{2} r_{t}^{2}=0$, and $x_{t}^{2} r_{t}^{2}=0$ for all $t$. Namely, let $t^{*}$ be the smallest index with $s_{t-1}^{2} x_{t}^{2}>0$ in the optimal solution of the follower picked by the leader. Then there exists $\ell<t^{*}$ with $s_{\ell-1}^{2}=0$, and $x_{\ell}^{2} \geq s_{\ell}^{2} \geq \cdots \geq s_{t^{*}-1}^{2}>0$, where the inequalities follow from the choice of $t^{*}$ and $\ell$, and from (11). Since the solution is optimal, we have $p_{\ell}^{2}+\sum_{\tau=\ell}^{t^{*}-1} h_{\tau}^{2}=p_{t^{*}}^{2}$. Let $\lambda=s_{t^{*}-1}^{2}$. We transform the optimal solution of the follower by decreasing $x_{\ell}^{2}$, and $s_{\ell}^{2}$ through $s_{t^{*}-1}^{2}$ by $\lambda$, and increasing $x_{t *}^{2}$ by $\lambda$. The new solution is still optimal for the follower, and this transformation has no impact at all on the feasibility or optimality of the solution of the leader.

We call a feasible solution of the bilevel lot-sizing problem extreme point solution if the follower's solution is an extreme point solution of ULSB (cf. Proposition 2). Let $Z_{t}$ denote the minimum cost incurred by backlogging a unit of pro- 
duction from period $t$ to a later period, that is $Z_{t}=\min _{u \geq t+1}\left(p_{u}+\sum_{v=t}^{u-1} g_{v}\right)$. Notice that $Z_{t}$ does not carry the fixed cost of production.

Lemma 4. The backlogged quantities $r_{t}$ in any optimal solution of ULSB satisfy

$$
\left(Z_{t}-p_{t}\right) r_{t} \leq f_{t}, \text { for } t=1, \ldots, n-1 \text {. }
$$

Proof. Let $(x, y, s, r)$ be any optimal solution of ULSB. We may assume that it satisfies the condition of Proposition 2. If $r_{t}=0$, then the inequality trivially holds. So assume that a positive amount of $r_{t}$ is backlogged in period $t$. Then, the total cost associated with this amount is at least $Z_{t} r_{t}$, which corresponds to the variable cost of backlogging it until some period $u$, and producing it in period $u$. On the other hand, if this amount were produced in period $t$ instead, then the associated cost would be at most $f_{t}+p_{t} r_{t}$. Hence, if $Z_{t} r_{t}>f_{t}+p_{t} r_{t}$, the solution is not optimal, because it would be cheaper to produce the $r_{t}$ products in period $t$ than in a later period.

A similar statement can be made about stocking costs. Let $S_{t}$ denote the minimum cost incurred by stocking a unit of production from some period $u<t$ until period $t$, that is $S_{t}=\min _{1 \leq u<t}\left(p_{u}+\sum_{v=u}^{t-1} h_{v}\right)$. Notice that $S_{t}$ does not carry the fixed cost of production.

Lemma 5. The stock levels $s_{t}$ in any optimal solution of ULSB satisfy

$$
\left(S_{t}-p_{t}\right) s_{t-1} \leq f_{t}, \text { for } t=2, \ldots, n .
$$

The proof is analogous to that of Lemma 4. We can apply these bounds to the follower's and to the leader's problem as well, and we will call them bound constraints. However, in our experience, these bounds are effective only when added to the follower's problem.

Proposition 1. The set of cuts (26) restricts the follower's solutions to extreme 
point solutions of ULSB:

$$
s_{t-1}^{2} \leq M\left(1-y_{t}^{2}-\beta_{t}^{2}\right), \quad t=1, \ldots, n,
$$

where we let $\beta_{n}^{2}=0$ to simplify notation, and $M=\sum_{t=1}^{n} d_{t}^{1}$.

Proof. We clearly have $r_{t}^{2} s_{t}^{2}=0$ for all $t$ in any optimal solution of the follower by Assumption 1. Now, if $r_{t}^{2}>0$, then $\beta_{t}^{2}=1$ by (16), and thus $s_{t-1}^{2}=y_{t}^{2}=0$ by (26), and then $x_{t}^{2}=0$ by (12). Likewise, if $x_{t}^{2}>0$, then $y_{t}^{2}=1$ by (12), whence $s_{t-1}^{2}=\beta_{t}^{2}=0$ by (26), and then $r_{t}^{2}=0$ by (16). Finally, if $s_{t-1}^{2}>0$, then $y_{t}^{2}=\beta_{t}^{2}=0$ by (26), and thus $x_{t}^{2}=r_{t}^{2}=0$ by (12) and (16), respectively.

Notice that $s_{t-1}^{2} \geq 0$ and (26) imply $y_{t}^{2}+\beta_{t}^{2} \leq 1$.

Finally, we mention that the complete linear description of ULSB provided in [15] cannot be applied directly to strengthening the linear formulation of $O P^{2}$, because all the inequalities involve terms of the form $\delta_{k, \ell} y_{t}$, which are nonlinear if the $\delta_{t}$ are variables instead of constants.

\section{Computational experiments}

Computational experiments were conducted to evaluate and compare the efficiency of 10 variants of the proposed MIP models:

MIP-N: formulation (21).

MIP-NB: MIP-N + bound constraints (24) and (25) added to the follower's problem.

MIP-NC: MIP-N + cuts (26) added to the follower's problem.

MIP-NCB: MIP-NC + bound constraints (24) and (25) added to the follower's problem.

MIP-F: formulation (23). 
MIP-FB: MIP-F + bound constraints (24) and (25) added to the follower's problem.

MIP-FC: MIP-F + cuts (26) added to the follower's problem.

MIP-FCB: MIP-FC + bound constraints (24) and (25) added to the follower's problem.

The models were implemented in the Mosel programming language of FICO Xpress [13] with default preprocessing and cut generation settings. The bound constraints and valid cuts were added to the MIP model before optimization was started in those variants using them. The experiments were run on an Intel Xeon X5650 2.67GHz computer under a Debian 6.0 operating system. The time limit was set to 1200 seconds for a single run on each instance.

A set of random problem instances was generated with five different problem sizes, $n \in\{10,20,30,40,50\}$. For each $n, 100$ problem instances were generated giving a total of 500 problem instances altogether. The parameters were randomized as follows:

$$
\begin{array}{rlll}
f_{t}^{1} \leftarrow U[100,200] & p_{t}^{1} \leftarrow U[1,5] & h_{t}^{1} \leftarrow U[2,20] & g_{t}^{1} \leftarrow U[4,40] \\
f_{t}^{2} \leftarrow U[250,1000] & p_{t}^{2} \leftarrow U[2,10] & h_{t}^{2} \leftarrow U[1,10] & g_{t}^{2} \leftarrow U[2,20] \\
d_{t} \leftarrow U[0,100] & & &
\end{array}
$$

where $U[a, b]$ is the uniform random distribution over the integers in interval $[a, b]$.

Table 2 presents the experimental results. All figures in the table are combined results over the instances with the given problem size. Column opt displays the number of instances solved to optimality out of 100 using the given model. Columns UB gap (LB gap) contain the maximum and average upper (lower) bound gaps in percent, respectively. For each instance $I$, the upper bound gap was computed as $100 \frac{U B(I)-L B^{*}(I)}{U B(I)}$, where $U B(I)$ is the upper bound found by the given MIP model, and $L B^{*}(I)$ is the best lower bound known for the instance, found by any of 
Table 2: Experimental results with MIP-N.

\begin{tabular}{|c|c|c|c|c|c|c|c|c|}
\hline & \multirow[t]{2}{*}{ opt } & \multicolumn{2}{|c|}{ LB gap (\%) } & \multicolumn{2}{|c|}{ UB gap (\%) } & \multicolumn{2}{|c|}{ time (sec) } \\
\hline & & & $\max$ & avg & $\max$ & avg & $\max$ & avg \\
\hline \multirow{5}{*}{$\begin{array}{l}Z 1 \\
\hat{e}^{\prime} \\
\sum\end{array}$} & $n=10$ & 100 & 0.00 & 0.00 & 0.00 & 0.00 & 0.49 & 0.16 \\
\hline & $n=20$ & 100 & 0.00 & 0.00 & 0.00 & 0.00 & 9.91 & 1.14 \\
\hline & $n=30$ & 100 & 0.00 & 0.00 & 0.00 & 0.00 & 188.00 & 16.75 \\
\hline & $n=40$ & 88 & 17.36 & 0.89 & 16.99 & 0.47 & 1200.44 & 329.86 \\
\hline & $n=50$ & 53 & 15.09 & 2.91 & 12.38 & 1.88 & 1200.90 & 749.97 \\
\hline \multirow{5}{*}{$\begin{array}{l}Z_{1}^{n} \\
\hat{\rho}^{\prime}\end{array}$} & $n=10$ & 100 & 0.00 & 0.00 & 0.00 & 0.00 & 0.53 & 0.16 \\
\hline & $n=20$ & 100 & 0.00 & 0.00 & 0.00 & 0.00 & 13.83 & 1.19 \\
\hline & $n=30$ & 100 & 0.00 & 0.00 & 0.00 & 0.00 & 357.02 & 22.00 \\
\hline & $n=40$ & 90 & 19.68 & 0.79 & 16.99 & 0.46 & 1200.30 & 322.78 \\
\hline & $n=50$ & 59 & 14.41 & 2.51 & 11.85 & 1.94 & 1200.58 & 714.31 \\
\hline \multirow{5}{*}{$\begin{array}{l}Z_{Z}^{\prime} \\
\hat{Z}^{\prime} \\
\sum_{\Sigma}\end{array}$} & $n=10$ & 100 & 0.00 & 0.00 & 0.00 & 0.00 & 0.61 & 0.18 \\
\hline & $n=20$ & 100 & 0.00 & 0.00 & 0.00 & 0.00 & 8.41 & 1.20 \\
\hline & $n=30$ & 100 & 0.00 & 0.00 & 0.00 & 0.00 & 248.62 & 16.07 \\
\hline & $n=40$ & 92 & 16.99 & 0.60 & 17.82 & 0.46 & 1200.30 & 248.81 \\
\hline & $n=50$ & 51 & 15.19 & 2.77 & 11.81 & 1.88 & 1200.65 & 730.48 \\
\hline \multirow{5}{*}{$\begin{array}{l}n \\
z^{\prime} \\
\rho^{\prime} \\
\sum\end{array}$} & $n=10$ & 100 & 0.00 & 0.00 & 0.00 & 0.00 & 0.76 & 0.27 \\
\hline & $n=20$ & 100 & 0.00 & 0.00 & 0.00 & 0.00 & 8.82 & 1.54 \\
\hline & $n=30$ & 100 & 0.00 & 0.00 & 0.00 & 0.00 & 158.35 & 14.74 \\
\hline & $n=40$ & 96 & 17.02 & 0.46 & 16.99 & 0.44 & 1200.26 & 227.71 \\
\hline & $n=50$ & 65 & 12.11 & 1.97 & 11.51 & 1.83 & 1200.55 & 645.94 \\
\hline
\end{tabular}

the approaches. Similarly, the lower bound gap was calculated as $100 \frac{U B^{*}(I)-L B(I)}{U B^{*}(I)}$, with $U B^{*}(I)$ and $L B(I)$ defined analogously. Columns time present the maximum and the average computation times, respectively.

The results show that models MIP-N and MIP-NB outperform MIP-NC and MIP-NCB only on small instances up to 20 time periods. From $n=30$, MIPNCB dominates all other variants in the comparison in almost all aspects, except the maximum lower bound gap, where for $n=40$, MIP-NC performs slightly better, but it solves only 92 instances to optimality compared to 96 by MIP-NCB. This indicates the efficacy of the bound constraints in eliminating dominated (suboptimal) solutions of the follower. We can also observe that combining the bound 
Table 3: Experimental results with MIP-F.

\begin{tabular}{|c|c|c|c|c|c|c|c|c|}
\hline & \multirow[t]{2}{*}{ opt } & \multicolumn{2}{|c|}{ LB gap (\%) } & \multicolumn{2}{|c|}{ UB gap (\%) } & \multicolumn{2}{|c|}{ time (sec) } \\
\hline & & & $\max$ & avg & $\max$ & avg & $\max$ & avg \\
\hline \multirow{5}{*}{$\stackrel{\text { I }}{\stackrel{1}{\prime}}$} & $n=10$ & 100 & 0.00 & 0.00 & 0.00 & 0.00 & 0.83 & 0.35 \\
\hline & $=20$ & 100 & 0.00 & 0.00 & 0.00 & 0.00 & 21.75 & 2.52 \\
\hline & $n=30$ & 100 & 0.00 & 0.00 & 0.00 & 0.00 & 242.45 & 24.82 \\
\hline & & 90 & 18.53 & 1.03 & 14.45 & 0.37 & 1200.48 & 344.31 \\
\hline & $n=50$ & 41 & 28.85 & 5.66 & 12.67 & 1.90 & 01.00 & 868.70 \\
\hline \multirow{5}{*}{$\begin{array}{l}\frac{n}{I} \\
\rho^{\prime} \\
\sum\end{array}$} & $=10$ & 100 & 0.00 & 0.00 & 0.00 & 0.00 & 1.08 & 0.29 \\
\hline & 20 & 100 & 0.00 & 0.00 & 0.00 & 0.00 & 13.48 & 1.53 \\
\hline & $n=30$ & 100 & 0.00 & 0.00 & 0.00 & 0.00 & 222.35 & 22.56 \\
\hline & $n=40$ & 89 & 18.14 & 0.86 & 16.05 & 0.39 & 1200.39 & 315.48 \\
\hline & $n=50$ & 53 & 21.65 & 3.51 & 11.84 & 1.81 & 1200.70 & 773.40 \\
\hline \multirow{5}{*}{ 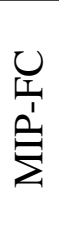 } & $=10$ & 100 & 0.00 & 0.00 & 0.00 & 0.00 & 1.02 & 0.43 \\
\hline & 20 & 100 & 0.00 & 0.00 & 0.00 & 0.00 & 16.64 & 1.92 \\
\hline & $n=30$ & 100 & 0.00 & 0.00 & 0.00 & 0.00 & 112.50 & 13.92 \\
\hline & $n=40$ & 90 & 15.61 & 0.71 & 13.67 & 0.34 & 1200.33 & 291.13 \\
\hline & $n=50$ & 45 & 24.30 & 5.01 & 14.78 & 1.91 & 1200.81 & 857.31 \\
\hline \multirow{5}{*}{$\begin{array}{l}0 \\
\text { 足 } \\
\hat{e}^{\prime} \\
\sum\end{array}$} & 10 & 100 & 0.00 & 0.00 & 0.00 & 0.00 & 1.14 & 0.47 \\
\hline & $n=20$ & 100 & 0.00 & 0.00 & 0.00 & 0.00 & 15.89 & 1.94 \\
\hline & $n=30$ & 100 & 0.00 & 0.00 & 0.00 & 0.00 & 103.94 & 11.67 \\
\hline & $n=40$ & 97 & 13.67 & 0.33 & 14.59 & 0.35 & 1200.26 & 224.12 \\
\hline & $n=50$ & 60 & 15.74 & 2.55 & 11.81 & 1.75 & 1200.59 & 743.18 \\
\hline
\end{tabular}

constraints (24) and (25), and the cuts (26) is really powerful on larger instances.

The results obtained by the four variants of MIP-F are summarized in Table 3. We can observe that each variant of MIP-F provides weaker lower bounds on average than the corresponding variant of MIP-N using more computation time in most cases. Likewise, MIP-F solves less instances to optimality than the corresponding variant of MIP-N in almost all cases. In fact, the LP relaxation of MIP-F is tighter, but the cutting planes of FICO Xpress are less effective on this formulation. On the other hand, MIP-F has better upper bound gaps on average in some cases. We conclude that no variant of MIP-N dominates any variant of MIP-F in all aspects, and vice versa, but MIP-N has an advantage in proving optimality, 
since cutting planes are more effective on this formulation than on MIP-F.

We have also tested further variants both for modeling the leader's, as well as the follower's constraints, but we got inferior results. For instance, the leader's constraints could be modeled by a shortest path formulation of ULSB (cf. Lemma 6). On the other hand, we have also tried to model the follower's optimal solutions as shortest paths in a network with arcs corresponding to regeneration intervals and with parametric arc lengths, but the computational results were weak.

\section{Conclusions}

In this paper we have developed exact solutions methods for the bilevel uncapacitated lot-sizing problem with backlogs. The novelty of our approach lies in the modeling of the optimality conditions of the follower by using a primal and a dual formulation for the same problem connected by a single equation, and thus we can avoid the use of extra binary variables to model complementarity conditions which is a standard technique in bilevel optimization.

Since by relaxing the integrality of the $y^{2}$ and $\beta^{2}$ variables in the definition of the mixed-integer set $O P_{e x t}^{2}$, the resulting linear system admits feasible solutions with fractional $y^{2}$ and $\beta^{2}$ values, a detailed polyhedral study of the the convex hull of $O P_{\text {ext }}^{2}$ is subject to future work. Such a study may be quite useful on its own, since the parametric solution of ULSB may have further important applications.

The capacitated version of the problem is even more difficult, even if the follower's problem is a constant capacity lot-sizing problem with backlogs (CCLSB). Although CC-LSB is polynomially solvable, to apply the technique of this paper, one needs an (extended) formulation for CC-LSB in which the demands occur in the objective function only. The extended formulation of [29] is not appropriate for our purposes, since the demands occur in the right hand side.

Finally, our approach may be suitable for solving other bilevel optimization problems where the follower's problem admits an extended formulation in which the parameters imposed by the leader occur only in the objective function, and the optimal solutions have nice structural properties. 


\section{Acknowledgments}

The authors are grateful to the anonymous referees for constructive comments that helped improve the content of the paper. This work has been supported by the Hungarian OTKA grant K76810, the NKTH grant OMFB-01638/2009. The work of Tamás Kis has been supported by the János Bolyai research grant $\mathrm{BO} / 00412 / 12 / 3$.

\section{Appendix A. Background in uncapacitated lot-sizing with backlogs}

In this section we recapitulate fundamental results on uncapacitated lot-sizing problems with backlogs (ULSB). The problem with a linear cost function can be stated as a mixed-integer linear program:

$$
\min \left\{\sum_{t=1}^{n}\left(p_{t} x_{t}+f_{t} y_{t}+h_{t} s_{t}+g_{t} r_{t}\right) \mid(A .2)-(A .6)\right\}
$$

where

$$
\begin{array}{ll}
x_{t}+\left(s_{t-1}-r_{t-1}\right)=\delta_{t}+\left(s_{t}-r_{t}\right), & t=1, \ldots, n \\
x_{t} \leq M y_{t}, & t=1, \ldots, n \\
s_{0}=s_{n}=r_{0}=r_{n}=0, & \\
x_{t}, s_{t}, r_{t}, \geq 0, & t=1, \ldots, n \\
y_{t} \in\{0,1\}, & t=1, \ldots, n
\end{array}
$$

In this formulation, $p_{t}, f_{t}, h_{t}, r_{t}$, and $\delta_{t}$ denote the marginal production cost, the fixed production cost, the marginal inventory holding cost, the marginal backlogging cost, and the demand in time period $t$, respectively; and $M=\sum_{t=1}^{n} \delta_{t}$ is a big constant. The variables $x_{t}, s_{t}$, and $r_{t}$ represent the production, the stocking and backlogging quantities, respectively, and the binary variables $y_{t}$ indicate whether there is production in period $t$ or not. Let $X^{B L}$ denote the set of feasible solutions 
of ULSB:

$$
X^{B L}=\left\{(x, y, s, r) \in \mathbb{R}_{+}^{n} \times\{0,1\}^{n} \times \mathbb{R}_{+}^{n+1} \times \mathbb{R}_{+}^{n+1} \mid(x, y, s, r) \text { satisfy (A.2)-(A.6) }\right\}
$$

Firstly, recall a basic structural property of ULSB:

Proposition 2. ([22]) The extreme points of $\operatorname{conv}\left(X^{B L}\right)$ are of the following structure: there exists $2 q$ indices $1=\ell_{1} \leq i_{1}<\ell_{2} \leq i_{2}<\cdots<\ell_{q} \leq i_{q} \leq \ell_{q+1}=n$ such that

- $x_{i_{j}}=\sum_{t=\ell_{j}}^{\ell_{j+1}-1} \delta_{t}$, and $y_{i_{j}}=1$ for $j=1, \ldots, q$.

- $x_{t}=0$ and $y_{t} \in\{0,1\}$ for $t \in\{1, \ldots, n\} \backslash\left\{i_{1}, \ldots, i_{q}\right\}$

- $r_{t}=\sum_{k=\ell_{j}}^{t} \delta_{k}$, and $s_{t}=0$ for $t \in\left\{\ell_{j}, \ldots, i_{j}-1\right\}$ for all $j=1, \ldots, q$.

- $s_{t}=\sum_{k=t+1}^{\ell_{j+1}-1} \delta_{k}$, and $r_{t}=0$ for $t \in\left\{i_{j}, \ldots, \ell_{j+1}-1\right\}$ for all $j=1, \ldots, q$.

Moreover, $\operatorname{conv}\left(X^{B L}\right)$ has $n-1$ extreme rays, one for each $t=1, \ldots, n-1$ :

$$
s_{t}=r_{t}=1, s_{j}=r_{j}=0 \text { for } j \neq t, x_{j}=y_{j}=0 \text { for all } j=1, \ldots, n \text {. }
$$

By this result, the extreme points of $\operatorname{conv}\left(X^{B L}\right)$ partition the interval $[1, \ldots, n]$ into regeneration intervals $\left[\ell_{j}, \ldots, \ell_{j+1}-1\right]$ for $j=1, \ldots, q$, between which there is no material flow (stock or backlog) in any direction. The following extended

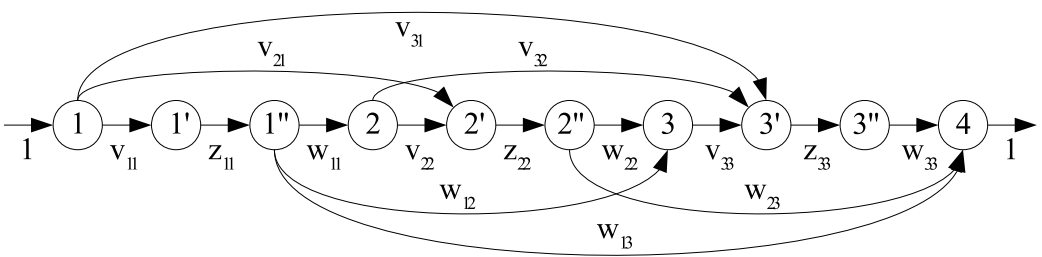

Figure A.1: The network of Lemma 6 for $n=3$.

formulation is based on solving ULSB by computing a shortest path in an appropriately defined network (see Figure A.1). The network consists of $3 n+1$ nodes. 
For each time period $t=1, \ldots, n$, there are 3 nodes, $t, t^{\prime}, t^{\prime \prime}$, and an extra node $(n+1)$. There is an arc from $t$ to $t^{\prime}$, from $t^{\prime}$ to $t^{\prime \prime}$, an arc from $t$ to $k^{\prime}$ for $t<k \leq n$, and an arc from $t^{\prime \prime}$ to $k$ with $t<k \leq n+1$. The associated flow variables are $v_{t t}$, $z_{t t}, v_{k t}$, and $w_{t, k-1}$, respectively. In addition, an extra variable $y_{t}$ is associated with $\operatorname{arc}\left(t^{\prime}, t^{\prime \prime}\right)$ for each $t$, to model the fixed cost of production in (A.1).

Lemma 6. ([22]) The optimum value of ULSB equals the optimum value of the following mathematical program.

$$
L^{S P}=\min \sum_{k=1}^{n}\left(\sum_{\ell=1}^{k-1} a_{k \ell} v_{k \ell}+p_{k} \delta_{k} z_{k k}+\sum_{\ell=k+1}^{n} b_{k \ell} w_{k \ell}\right)+\sum_{t=1}^{n} f_{t} y_{t}+\sum_{t=1}^{n-1}\left(h_{t}+g_{t}\right) \lambda_{t}
$$

subject to

$$
\begin{array}{ll}
\sum_{k=1}^{n} v_{k 1}=1, & \text { node } 1, \\
-v_{11}+z_{11}=0, & \text { node } 1^{\prime}, \\
-z_{11}+\sum_{\ell=1}^{n} w_{1 \ell}=0, & \text { node } l^{\prime \prime} \\
\sum_{k=t}^{n} v_{k t}-\sum_{k=1}^{t-1} w_{k, t-1}=0, & \text { node } t \text { for } t \geq 2, \\
-\sum_{\ell=1}^{t} v_{t \ell}+z_{t t}=0, & \text { node } t^{\prime} \text { for } t \geq 2, \\
-z_{t t}+\sum_{\ell=t}^{n} w_{t \ell}=0, & \text { node } t^{\prime} \text { for } t \geq 2, \\
z_{t t}-y_{t} \leq 0, & \text { for all } t, \\
y_{t} \leq 1, & \text { for all } t, \\
v_{k t}, z_{t t}, w_{k t}, y_{t}, \lambda_{t} \geq 0, & \text { for all } t,
\end{array}
$$

with $a_{k \ell}=p_{k} \delta_{\ell, k-1}+\sum_{t=\ell}^{k-1} g_{t} \delta_{\ell, t}$ for $1 \leq \ell<k \leq n$, and $b_{k \ell}=p_{k} \delta_{k+1, \ell}+\sum_{t=k}^{\ell-1} h_{t} \delta_{t+1, \ell}$ for $1 \leq k<\ell \leq n$, and $\delta_{k, \ell}=\sum_{t=k}^{\ell} \delta_{t}$ for $1 \leq k \leq \ell \leq n$. Moreover, $v, w, y$, and $z$ take integral values in any basic solution.

Clearly, if $h_{t}+g_{t} \geq 0$ for all $t$ (i.e., the optimum is finite), then the variables $\lambda_{t}$ can be dropped from the above formulation. Notice that all the demands are in the objective function. This formulation has $O\left(n^{2}\right)$ variables and $O(n)$ constraints.

In the same paper, Pochet and Wolsey describe the facility location based reformulation (FL). Let the variable $z_{k t}$ represent the amount of production in time period $k$ used to satisfy some of the demands in period $t$. 
Lemma 7. ([22]) The optimum value of ULSB equals the optimum value of the following mathematical program.

$$
L^{F L}=\min \sum_{k=1}^{n} \sum_{t=1}^{n} q_{k t} z_{k t}+\sum_{t=1}^{n} f_{t} y_{t}+\sum_{t=1}^{n-1}\left(h_{t}+g_{t}\right) \lambda_{t}
$$

subject to

$$
\begin{array}{ll}
\sum_{k=1}^{n} z_{k t}=\delta_{t}, & \text { for all } t \\
z_{k t}-\delta_{t} y_{k} \leq 0, & \text { for all } k, t \\
z_{k t} \geq 0,0 \leq y_{k} \leq 1, & \text { for all } k, t,
\end{array}
$$

where $q_{k t}=\left(p_{k}+h_{k}+\cdots h_{t-1}\right)$ if $k \leq t$ and $q_{k t}=\left(p_{k}+g_{t}+\cdots g_{k-1}\right)$ if $k>t$.

By moving the demands to the objective function, we get a reformulation with the same properties as the shortest path reformulation. However, the FL reformulation has $O\left(n^{2}\right)$ variables, and constraints.

[1] M. Albrecht. Supply Chain Coordination Mechanisms. Springer, 2010.

[2] S. Axsäter. Inventory Control. Springer, 2nd edition, 2006.

[3] I. Barany, T. J. Van Roy, and L. A. Wolsey. Strong formulations for multiitem capacitated lot-sizing. Management Science, 30:1255-1261, 1984.

[4] I. Barany, T. J. Van Roy, and L. A. Wolsey. Uncapacitated lot sizing: the convex hull of solutions. Mathematical Programming Study, 22:32-43, 1984.

[5] J. F. Bard. Coordination of a multidivisional organization through two levels of management. Omega, 11:457-468, 1983.

[6] G. Belvaux and L. A. Wolsey. Modelling practical lot-sizing problems as mixed-integer programs. Management Science, 47(7):993-1007, 2001.

[7] G. P. Cachon. Supply chain coordination with contracts. In A. G. de Kok and S. C. Graves, editors, Supply Chain Management: Design, Coordination and Cooperation, volume 11 of Handbooks in Operations Research and Management Science, pages 229-339. Elsevier, 2003. 
[8] B. Colson, P. Marcotte, and G. Savard. Bilevel programming: A survey. 4OR: A Quarterly Journal of Operations Research, 3:87-107, 2005.

[9] C. Cordier, H. Marchand, R. Laundy, and L. A. Wolsey. bc-opt: a branchand-cut code for mixed integer programs. Mathematical Programming, Ser. A, 86:335-353, 1999.

[10] S. Dempe. Foundations of Bilevel Programming. Kluwer, 2002.

[11] S. Dempe and A. B. Zemkoho. The bilevel programming problem: reformulations, constraint qualifications and optimality conditions. Mathematical Programming, Ser. A., in print, 2012.

[12] A. Drexl and A. Kimms. Lot-sizing and scheduling - survey and extensions. European Journal of Operational Research, 99(2):221-235, 1997.

[13] FICO-Xpress. Xpress Optimization Suite 7, 2009. http://www.fico.com/en/Products/ DMTools/Pages/FICO-XpressOptimization-Suite.aspx.

[14] A. Kovács, P. Egri, T. Kis, and J. Váncza. Inventory control in supply chains: alternative approach to a two-stage lot-sizing problem. International Journal of Production Economics, in print, 2012.

[15] S. Küçükyavuz and Y. Pochet. Uncapacitated lot-sizing with backlogging: the convex hull. Mathematical Programming, Ser. A, 118:151-175, 2009.

[16] H. Lee and S. Whang. Decentralized multi-echelon supply chains: Incentives and information. Management Science, 45(5):633-640, 1999.

[17] X. Li and Q. Wang. Coordination mechanisms of supply chain systems. European Journal of Operational Research, 179(1):1-16, 2007.

[18] Z. Lukač, K. Šorić, and V. Vojvodić Rosenzweig. Production planning problem with sequence dependent setups as a bilevel programming problem. European Journal of Operational Research, 187(3):1504-1512, 2008. 
[19] P. Marcotte and G. Savard. Bilevel programming: A combinatorial perspective. In D. Avis, A. Hertz, and O. Marcotte, editors, Graph Theory and Combinatorial Optimization, pages 191-217. Springer, 2005.

[20] R. A. Melo and L. A. Wolsey. Uncapacitated two-level lot-sizing. Operations Research Letters, 38(4):241-245, 2010.

[21] Y. Pochet. Mathematical programming models and formulations for deterministic planning problems. In M. Jünger and D. Naddef, editors, Computational Combinatorial Optimization, Lecture Notes in Computer Science, volume 2241, pages 57-111. Springer, 2001.

[22] Y. Pochet and L. A. Wolsey. Lot-size models with backlogging: Strong reformulations and cutting planes. Mathematical Programming, 40:317$335,1988$.

[23] Y. Pochet and L. A. Wolsey. Polyhedra for lot-sizing with Wagner-Whitin costs. Mathematical Programming Ser. A, 67:297-323, 1994.

[24] Y. Pochet and L. A. Wolsey. Production planning by mixed integer programming. Spinger, 2006.

[25] Y. Pochet and L. A. Wolsey. Single item lot-sizing with non-decreasing capacities. Mathematical Programming Ser. A, 121:123-143, 2010.

[26] E. Roghanian, S. J. Sadjadi, and M. B. Aryanezhad. A probabilistic bilevel linear multi-objective programming problem to supply chain planning. Applied Mathematics and Computation, 188:786-800, 2007.

[27] J-H. Ryu, V. Dua, and E. N. Pistikopoulos. A bilevel programming framework for enterprise-wide process networks under uncertainty. Computers and Chemical Engineering, 28:1121-1129, 2004.

[28] H. Stackelberg. The Theory of Market Economy. Oxford University Press, 1952. 
[29] M. van Vyve. Linear-programming extended formulations for the singleitem lot-sizing problem with backlogging and constant capacity. Mathematical Programming Ser. A, 108:53-77, 2006.

[30] A. Wagelmans, S. van Hoesel, and A. Kolen. Economic lot sizing: An $O(n \log n)$ algorithm that runs in linear time in the Wagner-Whitin case. $O p$ erations Research, 40:S145-S156, 1992.

[31] H. M. Wagner and T. M. Whitin. Dynamic version of the economic lot size model. Management Science, 5(1):89-96, 1958.

[32] W. Yang, L. Li, and S. Ma. Coordinating supply chain response-time: a bi-level programming approach. International Journal of Advanced Manufacturing Technology, 31:1034-1043, 2007.

[33] W. I. Zangwill. A backlogging model and a multi-echelon model of a dynamic economic lot size production system - a network approach. Management Science, 15(9):506-527, 1969.

[34] M. Zhang. Two-stage minimax regret robust uncapacitated lot-sizing problems with demand uncertainty. Operations Research Letters, 39:342-345, 2011. 